\begin{tabular}{|c|l|}
\hline Title & $\begin{array}{l}\text { Kaposi 's sarcoma associated herpesvirus encoded LANA associates with glucocorticoid receptor and enhances its } \\
\text { transcriptional activities }\end{array}$ \\
\hline Author(s) & $\begin{array}{l}\text { Togi, Sumihito; Nakasuji, Misa; Muromoto, Ryuta; Ikeda, Osamu; O kabe, Kanako; Kitai, Y uichi; Kon, Shigey uki; } \\
\text { Oritani, Kenji; Matsuda, Tadashi }\end{array}$ \\
\hline Citation & $\begin{array}{l}\text { Biochemical and biophysical research communications, 463(3), 395-400 } \\
\text { https://doi.org/10.1016/.bbrc.2015.05.080 }\end{array}$ \\
\hline Issue Date & 2015-07-31 \\
\hline Doc URL & http://hdl.handle.net/2115/62600 \\
\hline Rights & $\begin{array}{l}\text { O Elsevier, 2015. This manuscript version is made avail lable under the CC-BY-NC-ND 4.0 license } \\
\text { http://creativecommons.org/icenses/by-nc-nd/4.0/ }\end{array}$ \\
\hline Rights(URL) & http://creativecommons.org/icenses/by-nc-nd/4.0/ \\
\hline Type & article (author version) \\
\hline File Information & manuscript.pdf \\
\hline
\end{tabular}

Instructions for use 


\section{Kaposi's sarcoma-associated herpesvirus-encoded LANA associates with glucocorticoid receptor and enhances its transcriptional activities}

Authors: Sumihito Togi ${ }^{1}$, Misa Nakasuji ${ }^{1}$, Ryuta Muromoto ${ }^{1}$, Osamu Ikeda ${ }^{1}$, Kanako Okabe $^{1}$, Yuichi Kitai ${ }^{1}$, Shigeyuki Kon ${ }^{1}$, Kenji Oritani ${ }^{2}$ and Tadashi Matsuda ${ }^{1, *}$

Affiliation: ${ }^{1}$ Department of Immunology, Graduate School of Pharmaceutical Sciences Hokkaido University, Sapporo 060-0812, Japan, ${ }^{2}$ Department of Hematology and Oncology, Graduate School of Medicine, Osaka University, 2-2 Yamada-oka, Suita, Osaka 565-0871, Japan.

*Address for manuscript correspondence: Dr. Tadashi Matsuda, Department of Immunology, Graduate School of Pharmaceutical Sciences, Hokkaido University, Kita-Ku Kita 12 Nishi 6, Sapporo 060-0812, Japan TEL: 81-11-706-3243, FAX: 81-11-706-4990, E-mail: tmatsuda@pharm.hokudai.ac.jp 


\section{Abstract}

Kaposi's sarcoma-associated herpesvirus (KSHV)-encoded latency-associated nuclear antigen (LANA), which interacts with cellular proteins, plays a central role in modification of viral and/or cellular gene expression. Here, we show that LANA associates with glucocorticoid receptor (GR), and that LANA enhances the transcriptional activity of GR. Co-immunoprecipitation revealed a physical interaction between LANA and GR in transiently transfected $293 \mathrm{~T}$ and HeLa cells. In human B-lymphoma cells, LANA overexpression enhanced GR activity and cell growth suppression following glucocorticoid stimulation. Furthermore, confocal microscopy showed that activated GR was bound to LANA and accumulated in the nucleus, leading to an increase in binding of activated GR to the glucocorticoid response element of target genes. Taken together, KSHV-derived LANA acts as a transcriptional co-activator of GR. Our results might suggest a careful use of glucocorticoids in the treatment of patients with KSHV-related malignancies such as Kaposi’s sarcoma, primary effusion lymphoma, and multicentric Castleman disease.

Keywords: KSHV; LANA; GR; cross-talk; transcription 


\section{Introduction}

Kaposi's sarcoma-associated herpesvirus (KSHV), also known as human herpesvirus 8, is a human gamma herpes virus that mainly infects endothelial cells and B-lymphocytes $[1,2$, 3]. Numerous proteins encoded by the KSHV open reading frame during latency and lytic cycles of the virus have been identified, of which some are believed to transform the infected cells [1, 4]. Although KSHV was originally isolated from Kaposi's sarcoma (KS), the virus is also related to primary effusion lymphoma and multicentric Castleman disease $[5,6]$. Latency-associated nuclear antigen (LANA), a KSHV protein, is critical for the persistence of KSHV episomes, and functions in this capacity by tethering viral episomes to chromosomes during mitosis [7, 8, 9]. In addition, LANA interacts physically with various cellular proteins that regulate transcriptional activities, resulting in interfering with critical regulators of the cell cycle and enhancing anti-apoptotic effects $[10,11,12,13]$.

Glucocorticoid receptor (GR) is a member of the nuclear hormone receptor superfamily localized in the cytoplasm. Inactive GR is bound to a large protein complex including Hsp90 [14, 15]. When glucocorticoid (GC) binds to GR, Hsp90 dissociates, and the GC/GR complex translocates into the nucleus where it binds to specific palindromic sequences termed glucocorticoid response elements (GREs) [14, 15]. GC treatment of cells results in an increase or decrease in the transcription of a variety of target genes $[16,17]$. 
Because GCs induce growth inhibition and/or cell death in normal thymocytes, immature T-lymphocytes, and many malignant cells, GCs are major therapeutic agents for many types of autoimmune and allergic disorders as well as leukemias and lymphomas including KSHV-related malignancies [17, 18, 19]. However, many clinical data suggest that GCs may increase the incidence of KS in organ transplant recipients or exacerbate existing KS lesions [20-27]. It remains controversial whether GCs induce lytic viral gene expression in latently infected cells $[22,28]$, and the mechanisms underlying the clinical effects of GCs in $\mathrm{KS}$ are poorly understood despite their importance.

We previously reported that LANA associates with STAT3, and that LANA enhances the transcriptional activity of STAT3 [13]. However, ligand-bound GR forms a transactivating complex with STAT3 [29]. Thus, we clarified whether KSHV-encoded protein LANA influences GC-induced GR activation. In the present study, we found that LANA enhances transcriptional activation of GR. As the mechanism, we propose a physical interaction between LANA and GR. 


\section{Materials and Methods}

\section{Reagents and antibodies}

Dexamethasone (DEX) was purchased from Wako Chemicals (Osaka, Japan). RU486 was purchased from Sigma-Aldrich (St Louis, MO, USA). Expression vectors, MMTV-LUC, GRE-LUC, GR, epitope-tagged GR, LANA and LANA deletion mutants were described previously [13, 30, 31]. Anti-Myc, -GR and -Tublin antibodies were purchased from Santa Cruz Biotechnology (Santa Cruz, CA). Anti-FLAG M2 mAb, rabbit polyclonal anti-FLAG antibody were purchased from Sigma (St Louis, MO). Anti-LANA mAb was purchased from Advanced Biotechnologies Inc. (Maryland USA).

Cell culture, transfection and luciferase assays, Human cervix carcinoma cell line HeLa and human embryonic kidney carcinoma cell line 293T were maintained in Dulbecco's modified Eagle's medium (DMEM) containing $10 \%$ fetal bovine serum (FBS). HeLa cells were transfected using jetPEI (PolyPlus-transfection, Strasbourg, France) according to the manufacturer's instruction. 293T cells were transfected with the standard calcium precipitation protocol [30]. Human Burkitt's lymphoma DG75 B cells 
were grown in RPMI 1640 medium containing 10\% FBS. DG75 cells were nucleofected using Human B Cell Nucleofector Kit (Amaxa biosystems, Cologne, Germany) as described previously [13]. The stimulated cells were harvested and assayed for the luciferase activity using the Dual-Luciferase Reporter Assay System (Promega, Madison, WI) according to the manufacturer's instructions.

Immunoprecipitation, immunoblotting and DNA binding assay, Immunoprecipitation and Western blotting were performed as previously described [13]. The cells were harvested and lysed in lysis buffer (50 mM Tris-HCl, $\mathrm{pH} 7.4,0.15 \mathrm{M} \mathrm{NaCl}, 1 \% \mathrm{NP}-40$, $1 \mathrm{mM}$ sodium orthovanadate, $1 \mathrm{mM}$ phenylmethylsulfonyl fluoride, and $10 \mathrm{mg} / \mathrm{ml}$ each of aprotinin, pepstatin, and leupeptin). The immunoprecipitates from cell lysates were resolved on SDS-PAGE and transferred to an Immobilon filter (Millipore; Bedford, MA), which was then immunoblotted with antibodies. To measure GR DNA binding, cell extracts were treated with the immobilized GRE consensus oligonucleotide-Sepharose conjugate (Santa Cruz) as described previously [32].

Indirect Immunofluorescence microscopy, HeLa cells $\left(5 \times 10^{4}\right)$ seeded on a glass plate were fixed with $4 \%$ paraformaldehyde and reacted with respective antibodies. The cells 
were then reacted with FITC-conjugated anti-rabbit IgG or rhodamine-conjugated anti-mouse IgG (Chemicon) and observed under a confocal laser fluorescent microscope [31]. Images were obtained by using a Zeiss LSM 510 laser scanning microscope with an Apochromat x63/1.4 oil immersion objective and $x 4$ zoom. Nuclei were counterstained with 4', 6-diamidino-2-phenylindole (DAPI) (Wako, Osaka, Japan).

Cell viability assay, The numbers of viable DG75 cells after the indicated treatments $\begin{array}{lllll}\text { were } & \text { measured } & \text { using } & \text { WST-8 }\end{array}$ [2-(2-methoxy-4-nitrophenyl)-3-(4-nitrophenyl)-5-(2,4-disulfophenyl)-2H-tetrazolium, monosodium salt] assay (Cell Counting Kit-8; Wako Pure Chemicals). Briefly, $10 \mu 1$ of WST-8 solution was added to the cells in each well and incubated for $2 \mathrm{~h}$. The absorbances were measured at a test wavelength of $450 \mathrm{~nm}$ and a reference wavelength of $650 \mathrm{~nm}$ using a microplate reader (Bio-Rad, Hercules, CA).

Statistical analysis, The significance of differences between group means was determined by Student's t-test. 


\section{Results}

\subsection{LANA augments transcriptional activation of GR in $293 \mathrm{~T}$ and HeLa cells}

To assess the functional relevance between LANA and GR, we tested whether LANA affects GR-mediated transcriptional activation by transient transfection of 293T cells. 293T cells were transfected with GR and MMTV-LUC or GRE-LUC with or without LANA, and then luciferase activities were determined after treatment with dexamethasone (DEX). When $293 \mathrm{~T}$ cells were co-transfected GR and LANA, transcriptional activation of MMTV-LUC and GRE-LUC was augmented in a dose-dependent manner (Fig. 1A and B). We also tested the effects of LANA on endogenous GR activation in HeLa cells. HeLa cells were transfected with GRE-LUC with or without LANA, and then luciferase activities were determined after treatment with DEX. The same results were obtained because the transcriptional activation of GRE-LUC was augmented in a LANA dose-dependent manner (Fig. 1C). To confirm whether the DEX-induced GRE-LUC activation was mediated by endogenous GR, the cells were treated with RU486, a GR antagonist, under the same conditions. As a result, the GRE-LUC activity was completely blocked by RU486 after DEX stimulation in the presence or absence of LANA (Fig. 1C). Therefore, LANA specifically enhances GR transcriptional activities. 


\subsection{GR and LANA physically interact in vivo}

A mechanism consistent with the above data may be a DIRECT interaction between GR and LANA. To examine this possibility, we performed co-immunoprecipitation experiments in 293 cells that were transiently transfected with Myc-GR and FLAG-LANA expression vectors. The transfected $293 \mathrm{~T}$ cells were lysed and subjected to immunoprecipitation with an anti-FLAG antibody. The immunoprecipitates were then analyzed by western blotting with an anti-Myc antibody. As shown in Fig. 2A, the immunoprecipitates for GR clearly contained LANA proteins. To delineate the domains in LANA responsible for the protein-protein interactions with GR, co-immunoprecipitation experiments were performed with a series of mutant LANA proteins (Fig. 2B). As shown in Fig. 2C, mutants with deletion of the LANA central repeat domain (LANA-NC, $\Delta 333-981$ ) and C-terminal domain of LANA (LANA-C, 932-1162) interacted with GR, whereas the mutant with deletion of the N-terminal domain of LANA (LANA-N, 1-331) failed to interact with GR. Therefore, the C-terminal domain of LANA is required for the interaction with GR.

\subsection{LANA acts as a transcriptional activator of $G R$ in B-lymphocytes}

To understand the clinical significance of the molecular interaction between GR and LANA, we employed a KSHV-negative, Epstein-Barr virus-negative human B-lymphoma cell line, 
DG75, because B-lymphocytes are major targets of KSHV infection. The GR-LUC construct was co-transfected with LANA into DG75 cells (Fig. 3A), followed by assessment of reporter gene expression. LANA expression markedly enhanced DEX-induced GRE-LUC activities that were completely abolished by treatment with RU486 (Fig. 3스). We also examined the effects of LANA on GR-mediated growth suppression of DG75 B cells. As shown in Fig. 3므, ectopically expressed LANA significantly augmented DEX-induced growth suppression of DG75 cells. Therefore, LANA plays a critical role in the enhancement of GR activation in human B-lymphocytes.

\subsection{LANA influences the nuclear retention and DNA binding activity of GR}

To further characterize the interaction between GR and LANA, we determined the cellular location of the interaction. Ectopically expressed LANA localized to the nucleus in the presence or absence of DEX stimulation, while DEX stimulation induced translocation of endogenous GR into the nuclei of HeLa cells (Fig. 4A). In the absence of DEX stimulation, LANA expression tended to slightly enhance nuclear localization of GR. After DEX stimulation, we observed a significantly higher rate of cells with nuclear localization of GR in LANA-expressing HeLa cells than in control cells (Fig. 4A and B). The strong co-localization of LANA and GR suggests that activated GR interacts with LANA in the 
nucleus. Finally, we examined whether LANA expression affects the DNA-binding capacity of GR. A pull-down assay with consensus CRE oligonucleotides revealed that activated GR bound to the GRE sequence more efficiently in the presence of LANA (Fig. 4C). Therefore, LANA appears to enhance GR activities via augmentation of its DNA-binding activity through a DIRECT protein-protein interaction.

\section{Discussion}

In this study, we demonstrated a novel interaction between GR and KSHV-derived LANA.

LANA augmented the transcriptional activity of GR in cells. Furthermore, overexpression of LANA enhanced GR activity and GR-mediated growth suppression of human B-lymphocytes. As a possible mechanism, our results suggest that LANA-mediated enhancement of DEX-induced GR activation occurs via a physical interaction between LANA and GR in the nucleus.

LANA is a $222-232-\mathrm{kDa}$ nuclear protein expressed from open reading frame 73 of the KSHV genome $[4,7,8,9]$. LANA contains several conserved sequences including acidic-, proline-, and glutamine-rich domains as well as a zinc finger DNA-binding domain, leucine zipper, and potential nuclear localization signal. Its domain structure suggests that LANA plays a pivotal role in KSHV-infected cells. Indeed, LANA tethers the viral genome to the 
host cell genome, thus it is involved in long-term maintenance of viral episomal DNA in dividing cells $[7,8,9]$. In addition, LANA interacts with a variety of cellular proteins in the host. LANA can directly bind to and inhibit tumor suppressor p53 [10]. Through direct binding to glycogen synthase kinase $3 \beta$ and extracellular signal-regulated kinase (ERK), LANA enhances activation of ERK and c-myc. LANA also associates with hypophosphorylated retinoblastoma protein, and transactivates E2F-mediated promoter activities [11]. Our previous report also showed that LANA acts as a transcriptional activator for STAT3 [13]. In the present study, we identified GR as a new binding partner of LANA. The C-terminal portion of LANA (932-1162 aa) recognized the GR protein, and this region contains the DNA-binding domain of the LANA protein. After DEX stimulation, GRE-LUC activity was significantly enhanced and DG75 cell viability was decreased by LANA binding to GR. Some reports indicate that the incidence of KS is clinically increased by GCs [20-27]. The involvement of GR-mediated signals in KSHV-related malignancies will clarify the significance of LANA/GR interactions. It is also noteworthy that a recent study has indicated the LANA-nuclear RNA interaction through both $\mathrm{N}$ - and C-termini of LANA domain [33]. Similarly, the cellular non-coding RNA, growth arrest-specific transcript 5 (GAS5) has been demonstrated to interact GR directly via the DNA-binding domain of GR and represses GR activity by acting as a decoy GRE [34]. Therefore, these non-coding 
$\underline{\text { RNAs may bridge the LANA association with GR. Further detailed analysis will be required }}$

on this issue.

KSHV has a biphasic life cycle consisting of a life-long latent phase and a transient lytic reactivation phase $[1, \underline{35}]$. Only limited regions are activated in KSHV during latent infection. In contrast, the full repertoire of viral genes is activated in a temporally regulated manner during the lytic phase $[1,4]$. Two major KSHV products, LANA and RTA (replication and transcription activator), are believed to interact with each other, leading to switching between latent and lytic phases [ㅌ6]. RTA acts as an initiator and regulator of KSHV lytic DNA replication, and LANA represses RTA gene expression. Importantly, this inhibitory effect of LANA is partly regulated by its post-translational modification including arginine methylation, phosphorylation, and sumoylation $[4, \underline{37,38]}$. KSHV reactivation is also induced by certain stimuli such as viral co-infection as well as hypo- and hyper-oxidative responses $[1,4]$. Several reports indicate the involvement of GCs in KSHV reactivation [20-27]. GC treatment induces lytic viral gene expression in some, but not all, KSHV-infected B-lymphocyte lines. Therefore, LANA/GR interactions appear to enhance transition from latent to lytic phases during GC treatment.

In acquired immunodeficiency syndrome-related KS, highly active antiretroviral therapy is associated with a decrease in the size and number of lesions, leading to extension of the 
time to treatment failure $[\underline{39,40]}$. For patients with advanced KS, liposomal anthracyclines and taxane paclitaxel are clinically available. Although standard therapies are effective in the short-term, KS recurrence is common. Therefore, new therapeutic approaches are required. Recently, drugs have been developed to target LANA-mediated, angiogenetic, or anti-apoptotic signals. Our results may support the therapeutic potential of such LANA-targeting strategies. 


\section{Acknowledgments}

This study was supported in part by Grant-in-Aid for scientific research from Ministry of Education, Culture, Sports, Science and Technology of Japan. 


\section{References}

[1] E. A. Mesri, E. Cesarman, C. Boshoff, Kaposi's sarcoma and its associated herpesvirus, Nat. Rev. Cancer 10 (2010) 707-719.

[2] P. A. Carroll, E. Brazeau, M. Lagunoff, Kaposi's sarcoma-associated herpesvirus infection of blood endothelial cells induces lymphatic differentiation, Virology 328 (2004) 7-18.

[3] L. Chen, M. Lagunoff, Establishment and maintenance of Kaposi's sarcoma-associated herpesvirus latency in B cells, J. Virol. 79 (2005) 14383-14391.

[4] P. Purushothaman, T. Uppal, S. C. Verma, Molecular Biology of KSHV Lytic Reactivation, Viruses. 7 (2015) 116-153.

[5] P. S. Moore, Y. Chang, Detection of herpesvirus-like DNA sequences in Kaposi's sarcoma in patients with and without HIV infection, N. Engl. J. Med. 332 1181-1185.

[6] J. Soulier, L. Grollet, E. Oksenhendler, et al., Kaposi's sarcoma-associated herpesvirus-like DNA sequences in multicentric Castleman's disease. Blood 86 (1995) 
$1276-1280$.

[7] W. Zhong, H. Wang, B. Herndier, et al., RestricteDexpression of Kaposi sarcoma-associated herpesvirus (human herpesvirus 8) genes in Kaposi sarcoma. Proc. Natl. Acad. Sci. U S A. 93 (1996) 6641-6646.

[8] R. Sarid, O. Flore, R. A. Bohenzky, et al., Transcription mapping of the Kaposi's sarcoma-associated herpesvirus (human herpesvirus 8) genome in a body cavity-based lymphoma cell line (BC-1). J. Virol. 72 (1998) 1005-1012.

[9] M. E. Ballestas, P. A. Chatis, K. M. Kaye, Efficient persistence of extrachromosomal KSHV DNA mediated by latency-associated nuclear antigen. Science 284 (1999) 641-644.

[10] J. Friborg, W. Kong, M. O. Hottiger, et al., p53 inhibition by the LANA protein of KSHV protects against cell death. Nature 402 (1999) 889-894.

[11] S. A. Radkov, P. Kellam and C. Boshoff, The latent nuclear antigen of Kaposi sarcoma-associated herpesvirus targets the retinoblastoma-E2F pathway and with the oncogene Hras transforms primary rat cells. Nat. Med. 6 (2000) 1121-1127.

[12] M. Fujimuro, F. Y. Wu, C. ApRhys, et al.,A novel viral mechanism for dysregulation of beta-catenin in Kaposi's sarcoma-associated herpesvirus latency. Nat. 
Med. 9 (2003) 300-306.

[13] R. Muromoto, K. Okabe, M. Fujimuro, et al., Physical and functional interactions between STAT3 and Kaposi's sarcoma-associated herpesvirus-encoded LANA. FEBS Lett. 580(2006) 93-98.

[14] Ratman D, Vanden Berghe W, Dejager L, et al., How glucocorticoid receptors modulate the activity of other transcription factors: a scope beyond tethering.Mol Cell Endocrinol. 2013 Nov 5;380(1-2):41-54

[15] J. A. Gustafsson, A. C. Wikström, M. Denis, The non-activated glucocorticoid receptor: structure and activation, J Steroid Biochem. 34 (1989) 53-62.

[16] M. Karin, New twists in gene regulation by glucocorticoid receptor: is DNA binding dispensable? Cell.;93 (1998) 487-90.

[17] J. Saklatvala, Glucocorticoids: do we know how they work? Arthritis Res. (2002):146-150.

[18] R. A. Quax, L. Manenschijn, J. W. Koper, et al., Glucocorticoid sensitivity in health and disease, Nat. Rev. Endocrinol. 9 (2013) 670-686.

[19] Y. B. Chen, A. Rahemtullah, E. Hochberg, Primary effusion lymphoma, Oncologist 12 (2007) 569-576. 
[20] L. Corda, D. Benerecetti, M. Ungari, et al., Kaposi's disease and sarcoidosis, Eur. Respir. J. 9 (1996) 383-385.

[21] D. Farge, Kaposi's sarcoma in organ transplant recipients. The Collaborative Transplantation Research Group of Ile de France, Eur. J. Med. 2 (1993) 339-343.

[22] P. S. Gill, C. Loureiro, M. Bernstein-Singer, et al., Clinical effect of glucocorticoids on Kaposi sarcoma related to the acquired immunodeficiency syndrome (AIDS), Ann. Intern. Med. 110 (1989) 937-940.

[23] E. Gotti, G. Remuzzi, Post-transplant Kaposi's sarcoma. J. Am. Soc. Nephrol. 8 (1997) 130-137.

[24] I. Penn, 1The changing pattern of posttransplant malignancies. Transplant. Proc. 23 (1991) 1101-1103.

[25] P. L. Rady, E. Hodak, A. Yen, et al., Detection of human herpesvirus-8 DNA in Kaposi's sarcomas from iatrogenically immunosuppressed patients, J. Am. Acad.

Dermatol. 38 (1998) 429-437.

[26] A. Trattner, E. Hodak, M. David, et al., The appearance of Kaposi sarcoma during corticosteroid therapy. Cancer 72 (1993) 1779-1783.

[27] J. P. Vella, R. Mosher, M. H. Sayegh, Kaposi's sarcoma after renal transplantation. N. Engl. J. Med. 336 (1997) 1761. 
[28] J. P. Zoeteweij, A. S. Rinderknecht, D. A. Davis, et. al., Minimal reactivation of Kaposi's sarcoma-associated herpesvirus by corticosteroids in latently infected B cell lines, J. Med. Virol. 66 (2002) 378-383.

[29] Z. Zhang, S. Jones, J. S. Hagood, et al., STAT3 acts as a co-activator of glucocorticoid receptor signaling, J. Biol. Chem. 272 (1997) 30607-30610.

[30] T. Yamamoto, T. Matsuda, A. Junicho, et al., Cross-talk between signal transducer and activator of transcription 3 and estrogen receptor signaling, FEBS Lett. 486 (2000)143-148.

[31] R. Muromoto, M. Ishida, K. Sugiyama, et al., Sumoylation of Daxx regulates IFN-induced growth suppression of B-lymphocytes and the hormone receptor-mediated transactivation, J. Immunol. 177 (2006) 1160-1170.

[32] R. Muromoto, O. Ikeda, K. Okabe, et al., Epstein-Barr virus-derived EBNA2 regulates STAT3 activation, Biochem. Biophys. Res. Commun. 378 (2009) 439-443.

[33] M. Campbell, K. Y. Kim, P. C. Chang, et al., A lytic viral long noncoding RNA modulates the function of a latent protein, J. Virol. 88 (2014) 1843-1848. 
[34] T. Kino, D. E. Hurt, T. Ichijo T, et al., Noncoding RNA gas5 is a growth arrest-

and starvation-associated repressor of the glucocorticoid receptor, Sci. Signal. 3 (2010)

$\underline{\mathrm{ra} 8}$.

[35] L. A. Dourmishev, A. L. Dourmishev, D. Palmeri, et al., Molecular genetics of

Kaposi's sarcoma-associated herpesvirus (human herpesvirus-8) epidemiology and pathogenesis. Microbiol. Mol. Biol. Rev. 67 (2003)175-212.

[36] K. Lan, D. A. Kuppers, S. C. Verma, et al., Induction of Kaposi’s

sarcoma-associated herpesvirus latency-associated nuclear antigen by the lytic

transactivator RTA: A novel mechanism for establishment of latency. J. Virol. 79

(2005) 7453-7465.

[37] M. Campbell, P. C. Chang, S. Huerta, et al., Protein arginine methyltransferase 1-DIRECTed methylation of Kaposi sarcoma-associated herpesvirus latency-associated nuclear antigen, J. Biol. Chem. 287 (2012) 5806-5818.

[38] Campbell, M.; Izumiya, Y. Post-Translational Modifications of Kaposi's

Sarcoma-Associated Herpesvirus Regulatory Proteins-SUMO and KSHV. Front.

Microbiol. 3 (2012) 31. 
[39] R. Yarchoan, G. Tosato, R. F. Little, Therapy insight: AIDS-related

malignancies--the influence of antiviral therapy on pathogenesis and management, Nat

Clin. Pract. Oncol. (2005) 406-415.

[40] M. Berretta, R. Cinelli, F. Martellotta, et al., Therapeutic approaches to

AIDS-related malignancies.Oncogene. 22(2003) 6646-6659. 


\section{Figure Legends}

\section{Fig. 1. LANA augments DEX-induced transcriptional activation of GR}

(A) $293 \mathrm{~T}$ cells in a 12-well plate were transfected with MMTV-LUC (200 ng) together with GR (100 g) and/or increasing amounts of empty vector, expression vector for FLAG-LANA (200, $400 \mathrm{ng})$. Twenty-four hrs after transfection, the cells were stimulated with Dexamethasone (DEX; $100 \mathrm{nM}$ ) for an additional $12 \mathrm{hrs}$. The stimulated cells were harvested, and luciferase activities were measured. Total cell lysates (TCL) (1\%) was blotted with anti-FLAG or anti-GR antibody. (B) 293T cells in a 12-well plate were transfected with GRE-LUC (200 $\mathrm{ng})$ together with GR (100 g) and/or increasing amounts of empty vector, expression vector for FLAG-LANA (100, 200, $400 \mathrm{ng}$ ). Twenty-four hrs after transfection, the cells were stimulated with DEX (100 $\mathrm{nM})$ for an additional $12 \mathrm{hrs}$. The stimulated cells were harvested, and luciferase activities were measured. TCL (1\%) was blotted with anti-FLAG or anti-GR antibody. (C) HeLa cells in a 12-well plate were transfected with GRE-LUC (400 ng) and/or increasing amounts of empty vector, expression vector for FLAG-LANA (200, $400 \mathrm{ng})$. Twenty-four hrs after transfection, the cells were stimulated with DEX (100 nM) in the absence or presence of RU486 (100 $\mathrm{nM})$ for an additional $12 \mathrm{hrs}$. The stimulated cells 
were harvested, and luciferase activities were measured. TCL (1\%) was blotted with anti-FLAG antibody. The results are indicated as fold induction of luciferase activity from triplicate experiments, and the error bars represent the S.D. ${ }^{*} \mathrm{p}<0.01$.

\section{Fig. 2. GR and LANA physically interact in vivo}

(A) $293 \mathrm{~T}$ cells $\left(1 \times 10^{7}\right.$ cells $)$ were transfected with Myc-GR $(5 \mu \mathrm{g})$ together with or without FLAG-LANA (10 $\mu \mathrm{g})$. Forty-eight hrs after transfection, the cells were lysed, and immunoprecipitated with anti-FLAG antibody and immnoblotted with anti-Myc (upper panel) or anti-FLAG antibody (middle panel). Total cell lysates (TCL) (1\%) were blotted with anti-Myc or anti-FLAG antibody (lower panels).

(B) Domain structure of LANA and mutant fragments are schematically shown.

(C) $293 \mathrm{~T}$ cells $\left(1 \times 10^{7}\right.$ cells $)$ were transfected with Myc-GR $(5 \mu \mathrm{g})$ together with or without LANA-N (1-329), LANA-C (928-1162) and LANA-N+C ( $\Delta 330-927)(10 \mu \mathrm{g})$. Forty-eight hrs after transfection, the cells were lysed, immunoprecipitated with anti-Myc antibody and immunoblotted with anti-FLAG or anti-Myc antibody (upper panels). TCL (1\%) were blotted with anti-FLAG or anti-Myc antibody (lower panels).

Fig. 3. LANA acts as a transcriptional activator of GR in B-lymphocytes 
(A) Human Burkitt's lymphoma DG75 cells were nucleofected with $2.5 \mu \mathrm{g}$ of GRE-LUC, $2.5 \mu \mathrm{g}$ of pRL-TK and $2.5 \mu \mathrm{g}$ of an empty vector or expression vector for Myc-LANA by Nucleofector. Thirty-six hrs after transfection, the cells were stimulated with Dexamethasone (DEX; $50 \mathrm{nM})$ in the absence or presnce of RU486 (50 nM) for additional $8 \mathrm{hrs}$. The stimulated cells were harvested, and luciferase activities were measured. The results are indicated as fold induction of luciferase activity from triplicate experiments, and the error bars represent the S.D. ${ }^{*} p<0.01$. Total cell lysates (TCL) (1\%) was blotted with anti-Myc or anti-Tubulin antibody.

(B) DG75 cells were nucreofected as descrived the above. Twenty-four hrs after transfection, transfected cells were harvested and seeded into 96-well plates (1x10 /well). The cells were stimulated with increasing amounts of DEX for additional 24 hrs. The viable cells was evaluated by Cell Counting Kit-8. The value obtained for untreated cells ws set as $100 \%$ survival. Shown is a representative experiment, which was repeated at least three times with similar results, and the error bars represent the SD. $* * \mathrm{p}<0.01 * \mathrm{p}<0.05$.

\section{Fig. 4. LANA influences the nuclear retention and DNA binding activity of GR}

(A) HeLa cells in a 12-well plate were transfected with GR (400 ng) and/or Myc-LANA 
(400 ng). Thirty hrs after transfection, cells were treated with or without Dexamethasone (DEX; $50 \mathrm{nM}$ ) for $60 \mathrm{~min}$, and then fixed and reacted with rabbit anti-GR polyclonal and mouse anti-Myc antibody and visualized with FITC-conjugated anti-rabbit antibody (green) or rhodamine conjugated anti-mouse antibody (red). Nuclei were counterstained with DAPI (blue). Scale bars, $20 \mu \mathrm{m}$. (B) Quantitative analysis of the subcellular localization of GR. Approximately 100 cells were classified according to fluoreceine signals in the cytoplasm or nucleus. Results are representative of three independent experiments, and the error bars represent the SD. $* * p<0.01$. (C) $293 \mathrm{~T}$ cells $\left(1 \times 10^{7}\right.$ cells $)$ were transfected with Myc-GR $(5 \mu \mathrm{g})$ together with or without FLAG-LANA $(10 \mu \mathrm{g})$, and the cells were stimulated with Dexamethasone (DEX; 100 $\mathrm{nM}$ ) for $60 \mathrm{~min}$. To measure GR DNA binding, cell extracts were treated with the immobilized GRE consensus oligonucleotide-Sepharose conjugate. The precipitates were subjected to Western blot analysis using anti-GR antibody. Total cell lysates (TCL) (1\%) was blotted with anti-GR and anti-LANA antibodies. 
Figure 1
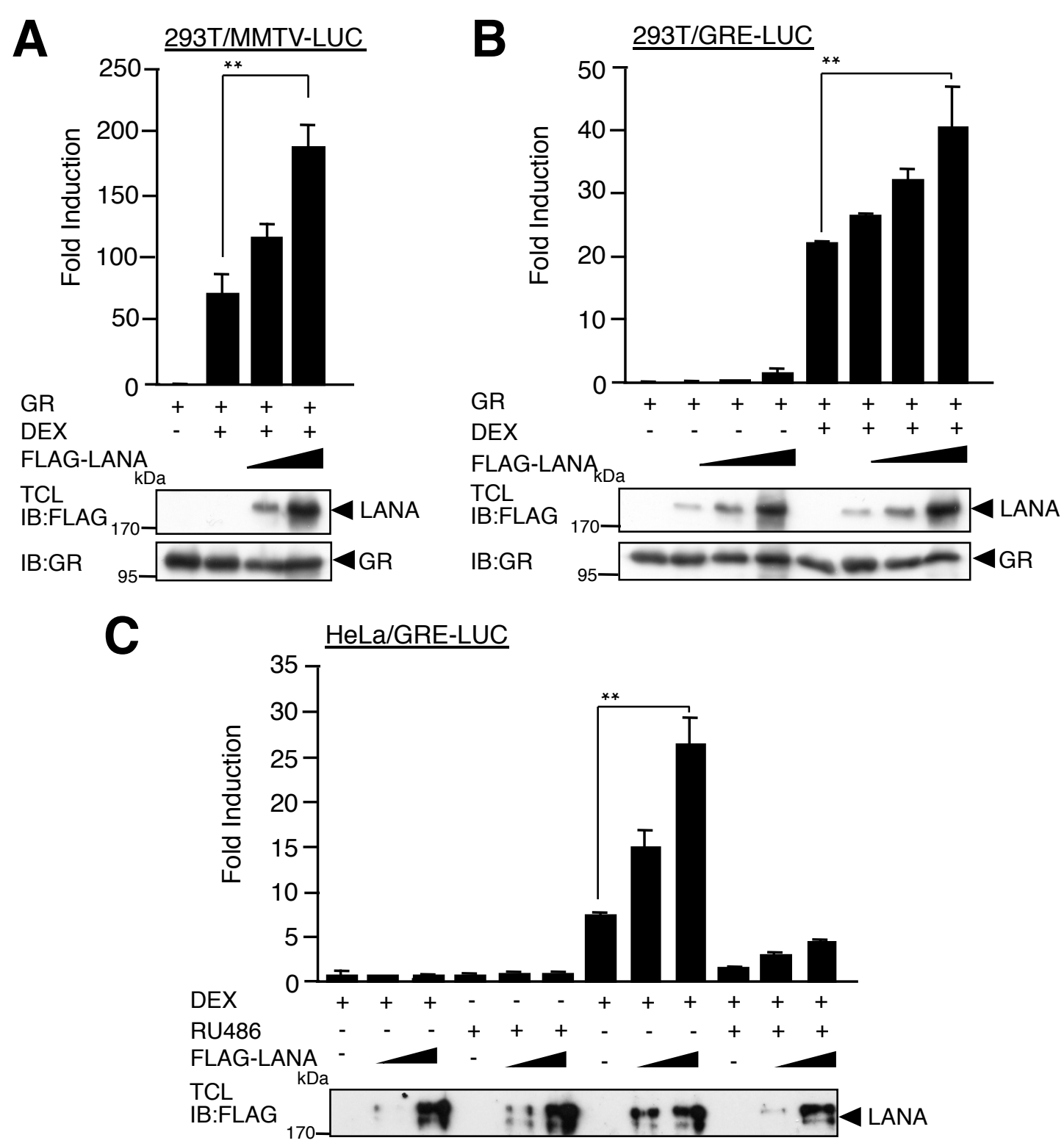


\section{Figure 2}
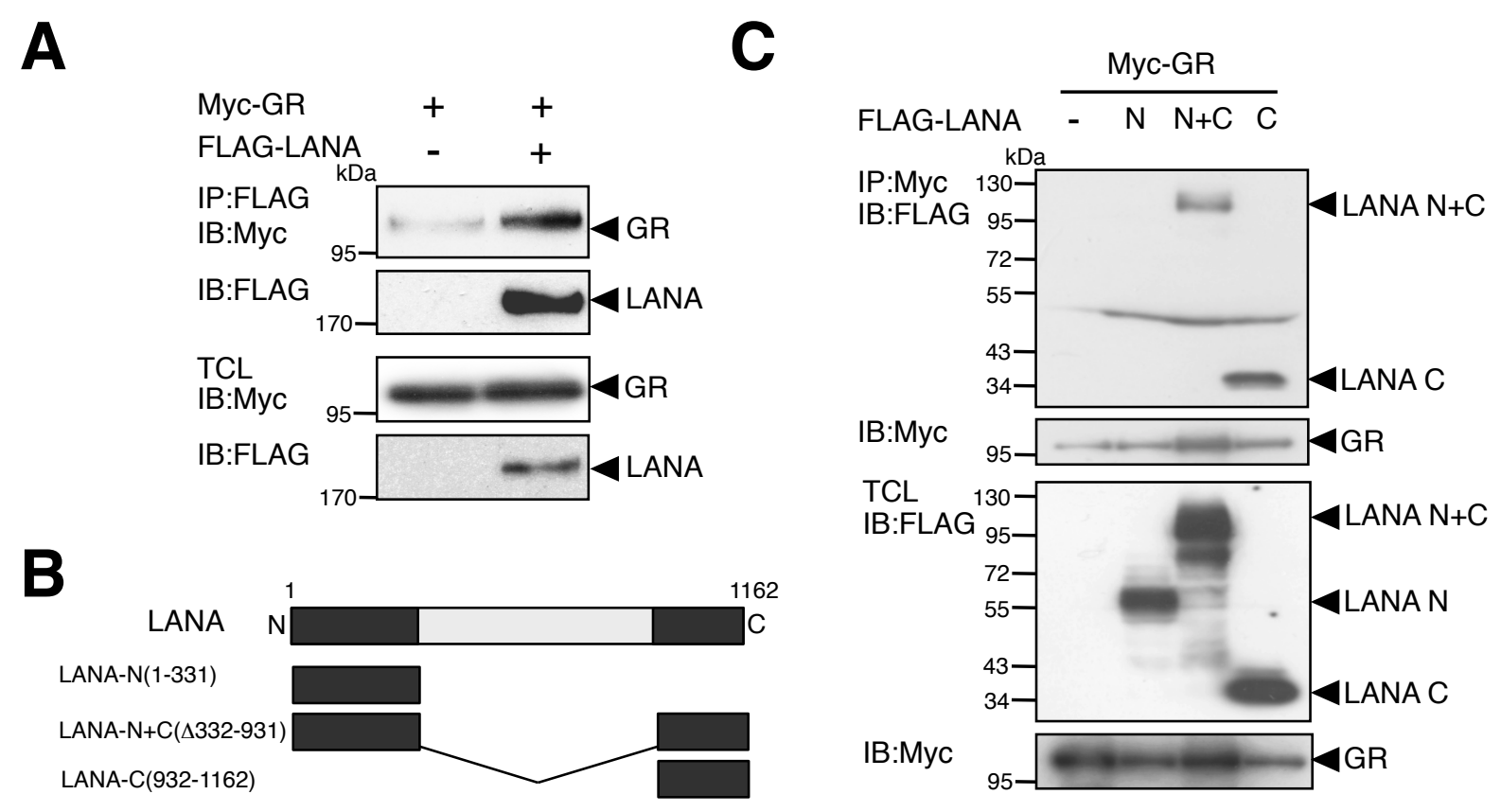
Figure 3

A

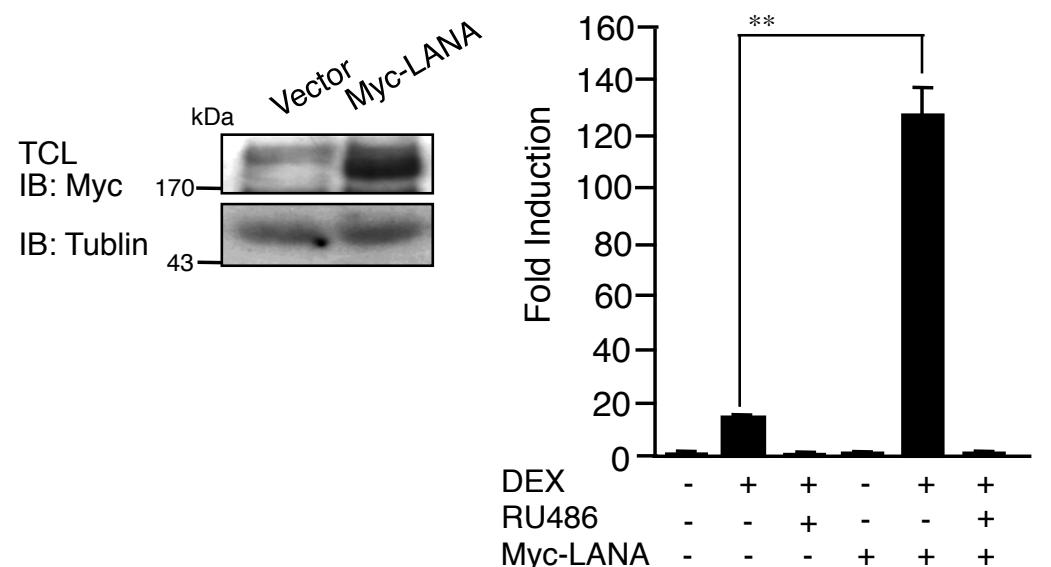

B

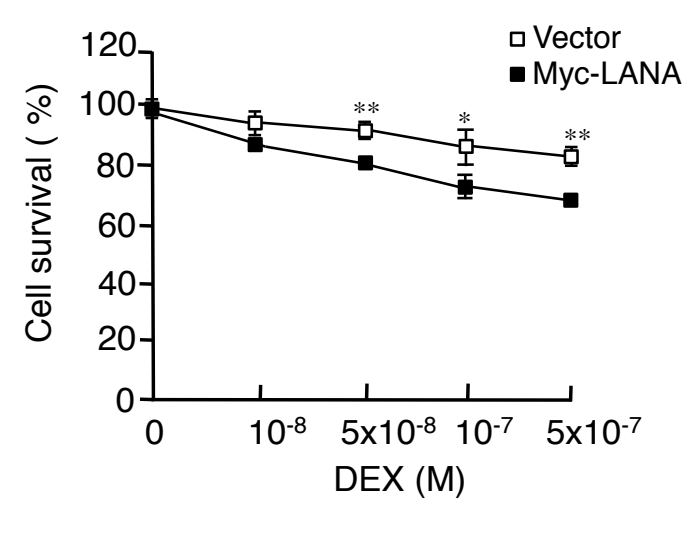


Figure 4

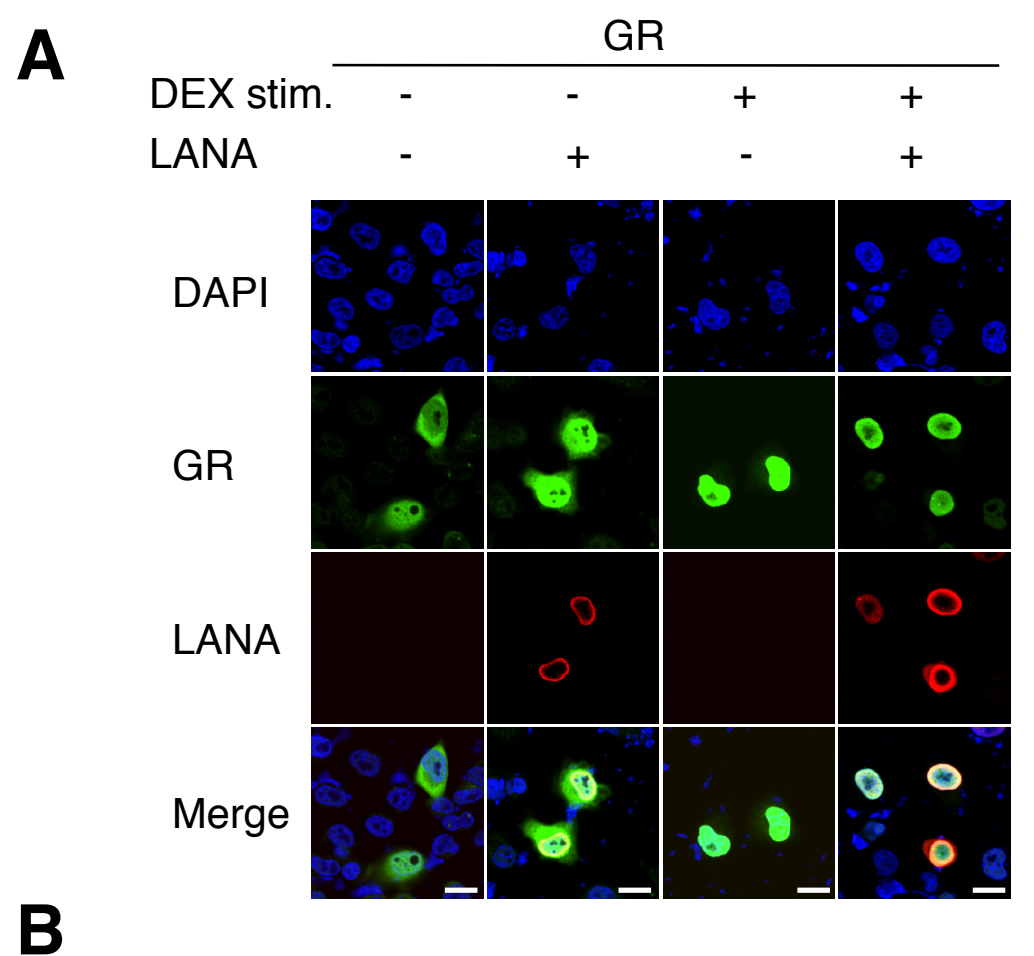

C
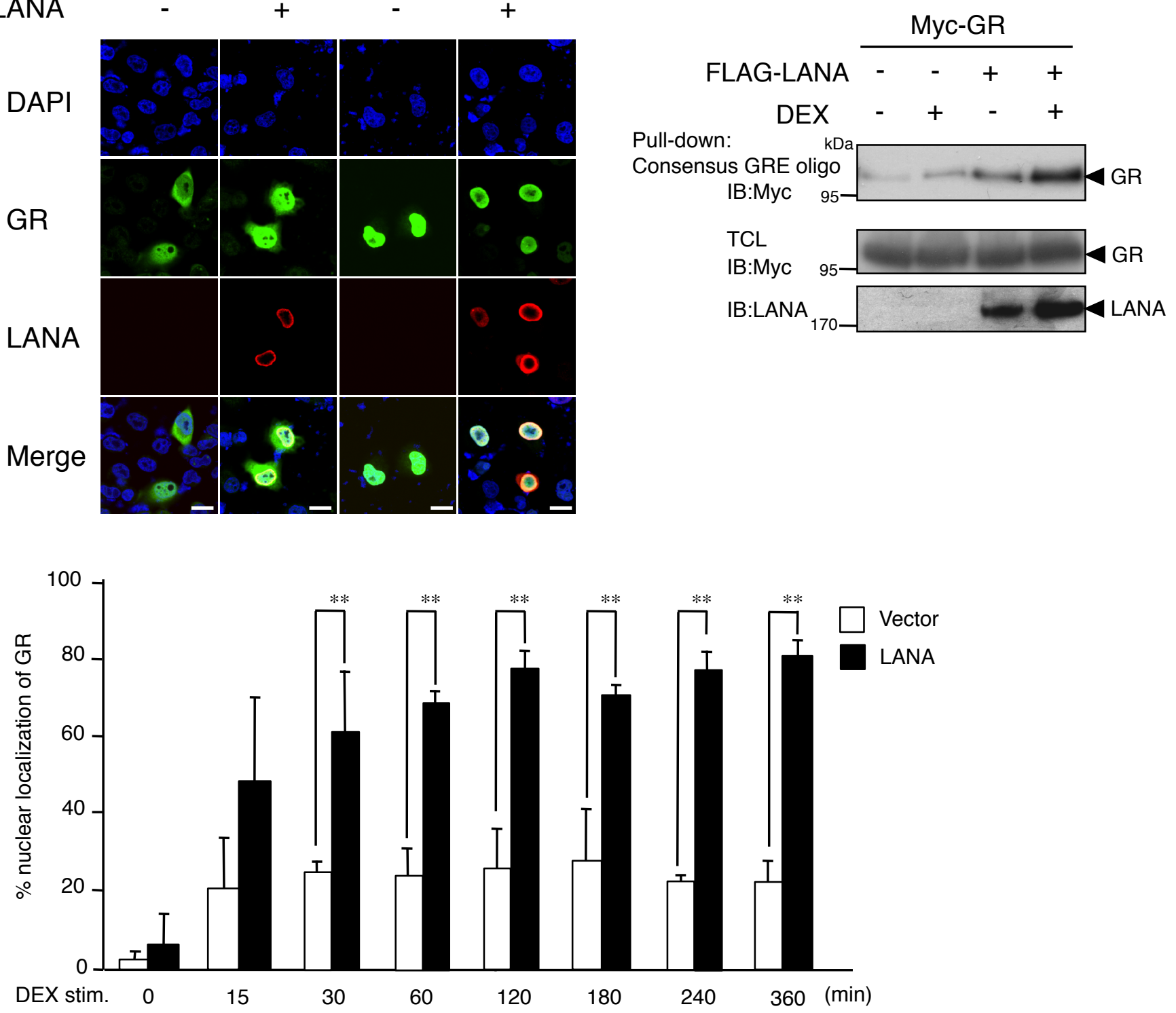